JGG 2021;69:233-237

doi: $10.36150 / 2499-6564-N 447$
Clinical Geriatrics - Reviews

\section{Characteristics of geriatric DM patients}

Raffaele Marfella

Department of Advanced Medical and Surgical Sciences, University of Campania "Luigi Vanvitelli" Naples, Italy; Italian Society of Gerontology and Geriatrics, Florence, Italy
Received: July 30, 2021

Accepted: September 16, 2021

\section{Correspondence}

\section{Raffaele Marfella}

Department of Advanced Medical and Surgical Sciences, University of Campania "Luigi Vanvitelli”, piazza Miraglia 2, 80138 Naples, Italy E-mail: raffaele.marfella@unicampania.it

How to cite this article: Marfella R. Characteristics of geriatric DM patients. Journal of Gerontology and Geriatrics 2021;69:233-237. https://doi. org/10.36150/2499-6564-N447

(C) Copyright by Società Italiana

di Gerontologia e Geriatria (SIGG)

\section{(c) (1) () (}

\section{OPEN ACCESS}

This is an open access article distributed in accordance with the CC-BY-NC-ND (Creative Commons Attribution-NonCommercial-NoDerivatives 4.0 International) license. The article can be used by giving appropriate credit and mentioning the license, but only for non-commercial purposes and only in the original version. For further information: https://creativecommons.org/licenses/by-nc-nd/4.0/deed.en

\section{RECOMMENDATIONS}

A. In the event of sudden changes in consciousness in geriatric patients, blood sugar should be assessed to exclude the presence of undetected, treatable DM causing cognitive impairment ${ }^{1,2}$.

B. Evaluation of glucose levels should be carried out in geriatric patients who have signs and symptoms of genitourinary system changes (polyuria, frequent urination, incontinence, mucocutaneous infections, recurrent cystitis) ${ }^{3}$.

C. When conducting a Comprehensive Geriatric Assessment of patients over 75 years of age, DM should be the object of screening 4,5 .

D. During Comprehensive Geriatric Assessment of the older diabetic patient, the autonomous nervous system evaluation should be carried out, including:

- changes in heart rate in response to forced breathing (Deep Breathing Test, in the supine position);

- changes in heart rate in response to the Valsalva maneuver (Valsalva test);

- changes in heart rate when changing from supine to orthostatic position (Active Stand Test);

- postural hypotension;

- assessment of sweating (reduced or absent);

- intestinal and bladder disorders ${ }^{4,5}$.

\section{STRENGTH OF THE RECOMMENDATIONS}

The quality of the evidence is high. Recommendations are supported by published evidence and best practice.

\section{SUPPORTING EVIDENCE}

See appendix.

\section{AREAS OF UNCERTAINTY AND FUTURE PERSPECTIVES}

In the future, it will be important to investigate the role that DM has in the onset of cognitive impairment and to identify factors predicting or mediating this risk. It will also be important to verify which hypoglycemic drug categories have the greatest capacity to reduce this risk. An important role will also be given by telemedicine to monitor the patient's glycemic values 
and in the rapid identification of red flags due to the onset of complications that often can be very dangerous.

\section{APPENDIX}

Geriatric patients do not usually present with typical DM symptoms in the initial stages of the disease (e.g., polyuria, polydipsia, polyphagia, and weight loss) ${ }^{4,5}$. Increasing the renal threshold for glucose reduces the likelihood of detecting glycosuria, making it difficult to identify polyuria and related symptomatic manifestations. Also, if secondary symptoms are present at DM onset in geriatric patients, they are usually masked by comorbid diseases.

\section{MASKING OF TYPICAL DM SYMPTOMS}

1 Polyuria rarely manifests in geriatric DM patients because of an increase in the renal glucose resorption threshold; if a geriatric patient experiences frequent urination, it is often underdiagnosed or wrongly attributed to genito-urinary diseases, which commonly occur in geriatric people (such as benign prostatic hyperplasia, incontinence, urinary tract infections), or to the use of diuretic drugs ${ }^{5}$.

2 Polydipsia is rare in geriatric patients. Although it has a role as a mechanism for protecting against hydroelectric loss caused by glycosuria, geriatric patients tend to experience reduced urine production because of a decrease in compensating for osmolarity. Polydipsia is also frequently associated with the presence of comorbid diseases (such as dysphagia, dementia, difficulties in staying hydrated without assistance), which make it difficult to identify and can lead to the start of a hyperosmolar coma. Rapid changes in consciousness are typical in DM patients with polydipsia due to hydro-electrolytic changes caused by increased blood glucose levels ${ }^{5}$.

3 Early-onset polyphagia is difficult to measure in geriatric patients. Specifically, patients tend to experience anorexia due to other changes (mouth abnormalities, gastrointestinal system deficits, associated pharmacological therapies, schizo-affective disorders, dementia, social isolation, poverty, poor quality care facilities) ${ }^{6}$.

\section{ROLE OF DIABETES COMPLICATIONS}

Complications due to DM dramatically reduce the quality of life of patients. This aspect is more evident in geriatric patients. Sensory alterations are the worst complications; these reduce patients' quality of life, mainly related to a loss of vision (due to cataract and retinopathy) and balance and gait disorders due to sensory and proprioceptive nervous deficits. These complications affect the patient's level of autonomy, resulting in a reduction in mobility, increasing geriatric syndromes, such as bed rest syndrome, and increasing the risk of falls ${ }^{7}$. Mucocutaneous infections are another frequent complication that can occur in DM patients. They can be highly disabling due to their frequent recurrence and difficulties in permanently resolving the infection ${ }^{8}$. The most frequent are mucocutaneous candidiasis (with all related manifestations), dermatophytosis, and bacterial infections, not least connected to the onset of diabetic foot complications. Another very common complication secondary to DM is the diabetic neuropathic pain syndrome ${ }^{9}$, characterized by paresthesia, dysesthesia, burning, or sensations of electric shock. The extent of this pain can be extremely severe and tends to worsen during the night, increasing the onset of sleep disorders. This symptom can become persistent and associated with allodynia, which can affect patients' quality of life, reducing their ability to carry out daily activities and affecting their mood. Pain can also cause social withdrawal and depression. Importantly, it is important to consider the role that micro- and macroangiopathic complications can have in DM patients' autonomy, which can greatly reduce their quality of life.

\section{UNSTABLE GLUCOSE LEVELS}

One of the main problems in managing geriatric DM patients is maintaining stable blood sugar levels. This should be taken seriously because:

1 geriatric individuals have a high risk of experiencing a hypoglycemic crisis, with an increased risk of falls and disability ${ }^{10}$;

2 hyperglycemic crises, which are associated with dehydration, are more likely to lead to hyperosmolar complications, which increases mortality risk ${ }^{10}$;

3 long-term elevated levels of fasting glucose in geriatric, non-insulin dependent DM patients are an independent risk factor for cardiovascular complications ${ }^{11}$.

Therefore, it is vital to optimize treatment in geriatric DM patients to consider all factors that may contribute to changes in glucose levels (concomitant drug therapies, poor drug adherence, inadequate diet, social isolation, poverty, dysphagia, gastrointestinal disorders, mouth abnormalities, neglect in long-term care facilities). To achieve this, a Comprehensive Geriatric Assessment of the patient is extremely important, with an emphasis on the role of regular blood sugar control and development of comorbidities (even at more frequent intervals), taking into account any changes in drug prescriptions due to a worsening of pre-existing comorbidities or the emergence of new acute factors, with more attention patient-centered outcomes ${ }^{12,13}$. 


\section{FREQUENT AUTONOMIC NEUROPATHY}

The risk of developing neuropathic complications in association with DM increases with age, regardless of the type of DM (T1DM, T2DM), making the management of geriatric patients more complex. Cardiac autonomic neuropathy is one of the most debilitating neuropathic complications in DM. It mostly manifests with abnormal control of heart rate and vagal tone. These changes may already be present at the time of diagnosis, especially in patients with T2DM. The condition plays a more important role in patients who already have other cardiovascular risk factors, such as arterial hypertension, smoking, dyslipidemia, or reduced glycemic control. This complication increases the risk of mortality of DM patients ${ }^{14,15}$. Reported prevalence rates vary in the literature, from 2 to $91 \%$ in T1DM and 25 to $75 \%$ in T2DM. The wide variability is due to differences in diagnostic criteria as well as underestimation and under-diagnosis. Numerous studies suggest that an under-diagnosis of cardiac autonomic neuropathy may increase the risk of intra- or perioperative complications, such as bleeding or cardiac arrest. Some authors suggest that cardiac autonomic neuropathy can also sometimes be seen in people with prediabetes. It has recently been proposed that the emergence of cardiac autonomic neuropathy dysfunction might be an independent predictive factor for diabetic nephropathy. Symptoms of cardiac autonomic neuropathy are directly related to the degree of disease progression. At early stages, reduced heart rate variability is more dominant, but tachycardia and reduced tolerance to physical exercise may appear as the disease progresses ${ }^{15}$.

Characteristically, heart rate does not vary with sleep, stress, or physical exercise, and there is a poor response to adenosine, suggesting a complete loss of neurological control, increasing the risk of cardiovascular mortality. Among the most serious manifestations that can occur at advanced stages, the worst are orthostatic hypotension and sympathetic denervation. These changes increase the occurrence of fainting, dizziness, falls, visual disturbances, and nocturnal hypertensive crises (due to abnormal sympathetic tone associated with the denervation process). These changes reduce the body's response to physical exercise, leading to more serious complications in geriatric DM patients, such as a reduction in overall physical activity, increased insulin resistance, and a tendency for social withdrawal. A meta-analysis found that diabetic cardiovascular autonomic neuropathy's development increases the risk of mortality approximately threefold, independently from complications associated with the risk of falls. Many studies have shown that this neuropathy is associated with silent ischemia. This is an important point, especially considering that geriatric DM patients often do not exhibit the classic symptoms of acute coronary disease. Gastroparesis is one of the most frequent and specific, and debilitating autonomic neuropathological changes to the gastrointestinal system. Gastroparesis is defined as delayed gastric emptying in the absence of mechanical obstruction. Many patients and healthcare professionals involved in diabetes care are not aware of this condition. Specialist centers that manage gastroparesis report a prevalence ranging from 10 to $30 \%$ in patients with T2DM, although pooled data suggest that $5 \%$ is a more realistic figure. From a pathophysiological point of view, chronic spikes in glucose levels cause damage to Cajal's cells and enteric neurons, which are responsible for the impairment of gastric emptying. Because of autonomic denervation, there is an alternating gastric emptying responsible for the symptomatic manifestation of this condition. Patients suffering from this condition most frequently complain about the following symptoms: early satiety, the prolonged feeling of abdominal fullness, swelling, nausea, vomiting, and abdominal pain. These symptoms often appear to be extremely vague and unspecific and frequently appear to be related to other diseases. The abnormal gastric mechanics can also lead to alterations in postprandial glucose blood levels, making hypoglycemic treatment difficult. Studies have repeatedly reported extreme fluctuations in blood glucose levels in some patients suffering from gastroparesis, which, in rare cases, results in ketoacidosis coma.

Also, hyperglycemia induced by gastric alteration may lead to further difficulties in gastric emptying. Symptoms caused by this complication often lead patients to take additional drugs and change diet and insulin therapy. Other events that are secondary to diabetic autonomic neuropathy involve the urinary, genital system ${ }^{3}$. The most frequent symptoms range from urinary urgency and nocturia to more debilitating symptoms such as incontinence and bladder stones ${ }^{16}$. Typical diabetic cystopathy is characterized by an irritating feeling of bladder fullness, increased bladder wall thickness, reduced bladder contraction, and increased postvoiding residue. The first disturbances to appear are sensitivity problems (which can manifest as overactive bladder syndrome), while parenchymal volume changes only appear later. Characteristic complaints are sex-specific. Around $50 \%$ of middle-aged DM women report urinary incontinence secondary to diabetic autonomic dysfunction (with an increased risk in women taking insulin); these figures are statistically less significant in diabetic males, given the frequent co-occurrence of benign prostatic hypertrophy, which may cause similar symptoms, although it has been demonstrated that DM men have a twofold risk of lower urinary tract disorders (such as 
frequent urination, strangury, dripping). More typically, there is a strong association between DM and erectile dysfunction ${ }^{17}$. This complication is often already present at the time of diagnosis. Prospective studies have shown that more than $50 \%$ of DM males will be affected by erectile dysfunction within ten years of DMonset. It can present as erectile dysfunction, retrograde ejaculation, or both. Another manifestation of diabetic autonomic neuropathy is dysfunction of the perspiration system. Sweating deficiency predisposes the skin to dryness which can lead to diabetic foot complications ${ }^{18}$.

\section{DM ONSET IN CONNECTION WITH SURGICAL OR OTHER STRESS}

It is important to conduct glucose monitoring during all types of hospital admissions (planned surgery, trauma, and acute diseases). Hyperglycemic stress can be a crucial opportunity to identify an increased risk of developing DM (gestational diabetes). All geriatric patients who exhibit abnormal glucose values during hospitalization for other diseases should be closely monitored considering that DM is often detected in connection with acute cardio- and cerebrovascular complications, and there is a high frequency of microvascular complications when people are diagnosed with DM ${ }^{19-21}$.

\section{Ethical consideration}

None.

\section{Acknowledgement}

None.

\section{Funding}

None.

\section{Conflict of interest}

The Author declares no conflict of interest.

\section{References}

1 Sinclair AJ, Paolisso G, Castro M, et al. European diabetes working party for older people 2011. Clinical guidelines for type 2 diabetes mellitus. Executive summary. Diabetes Metab 2011;37(Suppl 3):S27-S38. https://doi.org/10.1016/ s1262-3636(11)70962-4

2 van den Berg E, de Craen AJ, Biessels GJ, et al. The impact of diabetes mellitus on cognitive decline in the oldest of the old: a prospective population-based study. Diabetologia 2006;49:2015-2023. https://doi.org/10.1007/ s00125-006-0333-1

3 Brown JS, Wessells $H$, Chancellor MB, et al. Urologic complications of diabetes. Diabetes Care 2005;28:177185. https://doi.org/10.2337/diacare.28.1.177

4 Paolisso G. Pathophysiology of diabetes in elderly people. Acta Biomed 2010;81(Suppl 1):47-53.
5 Motta M, Bennati E, Ferlito L, et al. Diabetes mellitus in the elderly: diagnostic features. Arch Gerontol Geriatr 2006;42:101-106. https://doi.org/10.1016/j. archger.2005.05.001

6 Vischer UM, Perrenoud L, Genet C, et al. The high prevalence of malnutrition in elderly diabetic patients: implications for anti-diabetic drug treatments. Diabet Med 2010;27:918924. https://doi.org/10.1111/j.1464-5491.2010.03047.x

7 Yang $Y$, Hu X, Zhang Q, et al. Diabetes mellitus and risk of falls in older adults: a systematic review and meta-analysis. Age Ageing 2016;45:761-767. https://doi.org/10.1093/ ageing/afw140

8 Lima AL, Illing T, Schliemann S, et al. Cutaneous manifestations of diabetes mellitus: a review. Am J Clin Dermatol 2017;18:541-553. https://doi.org/10.1007/ s40257-017-0275-z

9 Javed S, Alam U, Malik RA. Burning through the pain: treatments for diabetic neuropathy. Diabetes Obes Metab 2015;17:1115-1125. https://doi.org/10.1111/dom.12535

10 Sircar M, Bhatia A, Munshi M. Review of hypoglycemia in the older adult: clinical implications and management. Can J Diabetes 2016;40:66-72. https://doi.org/10.1016/j. jcjd.2015.10.004

11 Freeman J. Management of hypoglycemia in older adults with type 2 diabetes. Postgrad Med 2019;131:241-250. https://doi.org/10.1080/00325481.2019.1578590

12 Muggeo M, Zoppini G, Bonora E, et al. Fasting plasma glucose variability predicts 10-year survival of type 2 diabetic patients: the Verona Diabetes Study. Diabetes Care 2000;23:45-50. https://doi.org/10.2337/diacare.23.1.45

13 Paolisso G, Sinclair AJ. Effective clinical practice for older people with type 2 diabetes. Glucose regulation is important but other strategies also need to be included. J Nutr Health Aging 2010;14:3-4. https://doi.org/10.1007/ s12603-010-0001-5

14 Dimitropoulos G, Tahrani AA, Stevens MJ. Cardiac autonomic neuropathy in patients with diabetes mellitus. World J Diabetes 2014;5:17-39. https://doi.org/10.4239/wjd. v5.i1.17

15 Spallone V, Ziegler D, Freeman R, et al. Cardiovascular autonomic neuropathy in diabetes: clinical impact, assessment, diagnosis, and management. Diabetes Metab Res Rev 2011;27:639-653. https://doi.org/10.1002/dmrr.1239

16 Jacobson AM, Braffett $\mathrm{BH}$, Cleary PA, et al. Relationship of urologic complications with health-related quality of life and perceived value of health in men and women with type 1 diabetes: the Diabetes Control and Complications Trial/ Epidemiology of Interventions and Complications (DCCT/ EDIC) cohort. Diabetes Care 2015;38:1904-1912. https:// doi.org/10.2337/dc15-0286

17 Wessells $\mathrm{H}$. Insights and interventions in diabetes associated erectile dysfunction. J Urol 2013;190:15-16. https:// doi.org/10.1016/j.juro.2013.04.041 
18 Shivaprasad C, Amit G, Anish K, et al. Clinical correlates of sudomotor dysfunction in patients with type 2 diabetes and peripheral neuropathy. Diabetes Res Clin Pract 2018;139:188-194. https://doi.org/10.1016/j. diabres.2018.03.004

19 Ali Abdelhamid Y, Kar P, Finnis ME, et al. Stress hyperglycaemia in critically ill patients and the subsequent risk of diabetes: a systematic review and meta-analysis. Crit Care 2016;20:301. https://doi.org/10.1186/ s13054-016-1471-6
20 Jivanji CJ, Asrani VM, Windsor JA, et al. New-onset diabetes after acute and critical illness: a systematic review. Mayo Clin Proc 2017;92:762-773. https://doi. org/10.1016/j.mayocp.2016.12.020

21 Moradi S, Keshavarzi A, Tabatabaee SM. Is stress hyperglycemia a predicting factor of developing diabetes in future? Exp Clin Endocrinol Diabetes 2015;123:614-616. https://doi.org/10.1055/s-0035-1559719

\author{
This statement is: \\ 囚 Recommendation (supported by published evidence) \\ $\square$ Best practice (supported by expert opinion)
}

Quality of the evidence (in the case of recommendation):
$\square$ Low
$\square$ Moderate
$\square$ High

